\title{
Indústria 4.0: Building Information Modelling no Custo de Construção Pública
}

\author{
Industry 4.0: Buildling Information Modelling in Public Construction Cost \\ Industria 4.0: Building Information Modelling sobre el Costo del Construcción Pública
}

Recebido: 04/01/2021 | Revisado: 05/01/2021 | Aceito: 10/01/2021 | Publicado: 12/01/2021

\author{
Daniele Moreira Santos \\ ORCID: https://orcid.org/0000-0002-9300-7994 \\ Centro Estadual de Educação Tecnológica Paula Souza, Brasil \\ E-mail: daniele.msantos@fco.net.br \\ Fabrício José Piacente \\ ORCID: https://orcid.org/0000-0001-8306-4541 \\ Centro Estadual de Educação Tecnológica Paula Souza, Brasil \\ E-mail: fjpiacente@yahoo.com.br
}

\begin{abstract}
Resumo
Na construção civil a Industria 4.0 desponta como um caminho natural para modernização do setor. Neste sentido o Building Information Modelling (BIM) se destaca ao promover a inovação na construção civil. O governo brasileiro seguindo o movimento mundial tem criado políticas de divulgação e incentivo a adoção do BIM. A expectativa do governo é aprimorar as estimativas de custos, reduzir o número de irregularidades na gestão de obras e evitar desvios de recursos financeiros do Estado. Mas como o conceito BIM pode contribuir para aumentar a confiabilidade das estimativas por meio da aplicação da modelagem 4D e 5D. Por meio de uma revisão da literatura e exploração de pesquisas publicadas, relatórios do governo, artigos e outras fontes, este trabalho tem objetivo entender como as etapas de quantifição e orçamentação da Estrátegia BIM BR pode contribuir para assertividade das estimativas de custos e redução de irregularidades vinculadas a orçamentação. Identificando quais são as irregularidades decorrentes de equívocos na estimativa de custo e as possíveis contribuições e limitações do processo 4D e 5D.
\end{abstract}

Palavras-chave: Building Information Modelling; Indústria 4.0; Custo de obras pública; BIM 4D; BIM 5D.

\begin{abstract}
In civil construction, Industry 4.0 emerges as a natural path to modernize the sector. In this sense, Building Information Modelling (BIM) stands out for promoting innovation in civil construction. The Brazilian government, following the worldwide movement, has created disclosure policies and encouraged the adoption of BIM. The government's expectation is to improve cost estimates, reduce the number of irregularities in the management of works, and avoid misuse of state financial resources. But how the BIM concept can contribute to increasing the reliability of the estimates through the application of 4D and 5D modeling. Through a literature review and exploration of published research, government reports, articles, and other sources, this work aims to understand how the steps of quantification and budgeting of the BIM BR Strategy can contribute to the assertiveness of cost estimates and reduction of irregularities linked to budgeting. Identifying the irregularities resulting from mistakes in the cost estimate and the possible contributions and limitations of the $4 \mathrm{D}$ and 5D process.
\end{abstract}

Keywords: Building information modelling; Industry 4.0; Public construction cost; BIM 4D; BIM 5D.

\section{Resumen}

En la construcción civil, la Industria 4.0 surge como un camino natural para modernizar el sector. En este sentido, Building Information Modelling (BIM) destaca por promover la innovación en la construcción civil. El gobierno brasileño, siguiendo el movimiento mundial, ha creado políticas de divulgación y alentado la adopción de BIM. La expectativa del gobierno es mejorar las estimaciones de costos, reducir la cantidad de irregularidades en la gestión de las obras y evitar el mal uso de los recursos financieros estatales. Pero cómo el concepto BIM puede contribuir a incrementar la confiabilidad de las estimaciones mediante la aplicación de modelos $4 \mathrm{D}$ y 5D. A través de una revisión de la literatura y la exploración de investigaciones publicadas, informes gubernamentales, artículos y otras fuentes, este trabajo tiene como objetivo comprender cómo los pasos de cuantificación y presupuestación de la estrategia BIM BR pueden contribuir al asertividad de las estimaciones de costos y la reducción de irregularidades. vinculado a la presupuestación. Identificar las irregularidades resultantes de errores en la estimación de costos y las posibles contribuciones y limitaciones del proceso 4D y 5D.

Palabras clave: Building information modelling; Industria 4.0; Costo de obras públicas; BIM 4D; BIM 5D. 


\section{Introdução}

A Industria 4.0 é caracterizada pela integração das diversas tecnologias tanto físicas quanto digitais gerando uma transformação na forma de se produzir. No entendimento de Ronaldo Fragoso (2018) essa transformação é grande e gera enormes impactos. Na indústria da construção civil como ramificação da Indústria 4.0 surge a Construção 4.0. Um dos conceitos que representa essa transformação no setor é o Building Information Modelling (BIM) (Alaloul et al., 2018).

Acompanhando a tendência mundial, o governo brasileiro tem desprendido esforços na divulgação e implantação do BIM, em particular nas contratações referente a obras públicas. Os editais de licitações, conforme Decreto n 9.377 passará a exigir a utilização do BIM no desenvolvimento do produto, agregando transparência e tornando assertivas as etapas de concepção e gerenciamento das obras (Sampaio Nardelli, 2018).

Pesquisas relacionadas ao BIM 4D e 5D apontam os benefícios da implantação da metodologia para obter maior eficiência no controle dos custos. Para Ministério da Indústria, Comércio Exterior e Serviços (MDIC) a metodologia tem como principais benefícios o aumento na precisão de projetos (especificação, quantificação e orçamentação) redução dos retrabalhos, desperdícios, prazos e custos (Brasil, 2018a)

Problema esse anualmente retratado pelo Tribunal de Contas da União (TCU) que apenas no 2017 por meio de fiscalizações de obras (Fiscobras) consolidou 94 empreendimentos de infraestrutura, $\mathrm{R} \$ 26,2$ bilhões de investimento, que apresentaram irregularidades classificadas de acordo com a gravidade dos achados.

Portanto, como a Industria 4.0, por meio do conceito BIM, pode aumentar a confiabilidade das estimativas de custos das obras públicas. BIM é um conceito de integração paramétrica de objetos alimentado por um repositório de informações que facilitam a interoperabilidade e o intercâmbio de informações (Miettinen \& Paavola, 2014).

A metodologia é dividida em dimensões "nD" e cada uma delas representa uma etapa do ciclo de vida do produto. As dimensões 4D (tempo) e 5D (custo) são os modelos paramétricos dos quais é possível extrair com maior grau de precisão, respectivamente, os quantitativos de serviços e insumos, e o orçamento (Eastman et al., 2011).

O objetivo deste artigo é compreender como o processo de quantificação e orçamentação proposto pelo Agência Brasileira de Desenvolvimento Industrial (ABDI) em conjunto com o MDIC na Estratégia BIM BR pode tornar assertivas as estimativas de custos das obras públicas reduzindo assim o número de irregularidades identificadas pelo TCU.

Para elaboração deste trabalho foi realizada revisão de literatura incluiu a exploração de relatórios do governo, artigos e outras fontes publicadas. O resultado permitiu identificar quais são as irregularidades decorrentes de equívocos na estimativa de custo e as possíveis limitações do processo de modelagem 4D e 5D.

\section{Fundamentos Teóricos}

\subsection{Industria 4.0 na construção civil}

A Industria 4.0 surge com o objetivo de integrar os processos de produção com as tecnologias e técnicas de informação. Desta forma torna-se possível definir todo o ciclo de vida de um produto, desde desenvolvimento, fabricação, uso, manutenção até a reciclagem, otimizando os recursos materiais e financeiros (Nowotarski; Paslawski, 2017).

$\mathrm{Na}$ construção civil, a Indústria 4.0 desponta como um caminho natural para aumentar a competitividade, produtividade e redução de custos, surgindo assim uma ramificação desta, chamada "Construção 4.0" ou "Canteiro 4.0" (Alaloul et al., 2018; Miyasaka, Minto Fabricio \& Paoletti, 2018).

Dentre os conceitos que unem tecnologias e serviços, o BIM ganha maior protagonismo na Construção 4.0 (Alaloul et al., 2018). Isso ocorre porque o BIM proporciona a interação de políticas, processos e tecnologias tornando-se uma metodologia que facilita o gerenciamento de projetos ao longo do ciclo de vida da edificação (Succar, 2009).

O BIM é um processo inteligente e em constante evolução, por isso apresenta diferentes fases de maturidade que são 
denominadas dimensões "nD" (Eastman et al., 2011). Atualmente existem sete dimensões: 2D (projeto), 3D (modelo do objeto), 4D (tempo), 5D (custo), 6D (operação), 7D (sustentabilidade) e 8D (segurança) (Smith, 2014a).

Nos últimos anos tem se observado um crescimento na adoção implementação do BIM por muitos países ao redor do mundo (Smith, 2014b). No Brasil a adoção do BIM ganhou destaque entre os anos 2014 e 2016 devido a hospedagem de grandes eventos como a Copa do Mundo da FIFA e dos Jogos Olímpicos (MC Grawh C, 2014; Smith, 2014b). Com a finalidade de promover a inovação da indústria da construção, principalmente no setor público, o governo brasileiro lançou em 2018 a estratégia BIM BR instituída pelo Decreto nº 9.377 (Brasil, 2018a).

\subsection{Problema}

No mundo assim como no Brasil, projetos no setor público são frequentemente afetados por excedentes de custo e cronograma (Flyvbjerg, Holm \& Buhl, 2002). Em muitos casos, o custo final do projeto supera o estimado durante o planejamento inicial e aprovado no início da construção (Shane et al., 2009).

Reconhecendo a dificuldade do setor público em finalizar obras de diferentes portes e complexidade, em 2006, o Brasil (2018b) constatou por meio de auditoria que de uma amostra de 400 obras inacabadas, um investimento de $\mathrm{R} \$ 3,5$ bilhões, as principais causas de paralisação foram: fluxo orçamentário/financeiro, problemas no projeto ou execução da obra e inadimplência do tomador/convenente.

Após a consolidação das fiscalizações realizadas ao longo de um período de 10 anos constatou que de um universo de 1.688 obras fiscalizadas, 1.331 apresentaram irregularidade relacionada ao sobrepreço/superfaturamento e 749 relacionadas a falha na composição dos custos (Brasil, 2018b).

Com a implementação do BIM, por meio da Estratégia BIM BR o governo brasileiro almeja aumentar a confiabilidade nas estimativas de custos com a redução de erros de compatibilidade. E com processos mais precisos de orçamentação e planejamento promover a diminuição de custos, riscos e economia dos recursos, resultando em uma maior transparência do processo licitatório/compra (Brasil, 2018a).

\subsection{BIM 4D (tempo) e 5D (custo)}

Na dimensão 4D é agregado tempo ao modelo 3D do projeto, o que permiti desenvolver uma simulação gráfica em tempo real do progresso da construção. A adição da quarta dimensão oferece uma oportunidade para avaliar a capacidade de construção e o planejamento do fluxo de trabalho de um projeto. O que proporciona aos colaboradores do projeto visualizar, analisar e comunicar problemas relacionados aos aspectos sequenciais, espaciais e temporais do progresso da construção. (Kamardeen, 2010; Smith, 2014a)

A integração do 5D ao modelo BIM permite a geração instantânea de orçamento de custos e representações financeiras genéticas do modelo em relação ao tempo. Isso reduz o tempo de levantamento de quantidades e estimativas de custos de semanas para minutos, melhora a precisão das estimativas, minimiza os incidentes de disputas por incompatibilidade dos arquivos do CAD (Kamardeen, 2010; Smith, 2014a)

\section{Metodologia}

A pesquisa é classificada como qualitativa e apresenta como característica principal o cunho exploratório. Como parte de um estudo maior sobre estimativas e o gerenciamento de custos foi realizada uma revisão da literatura para identificar os fatores que influenciam no crescimento do custo e paralisação das obras públicas.

A revisão de literatura incluiu a exploração de relatórios e publicações de pesquisa, relatórios do governo, artigos e outras fontes publicadas. Após a conclusão da revisão da literatura foi realizada uma análise dos dados levantados pelo TCU, 
por meio de fiscalizações de obras (Fiscobras) no ano de 2018, com o objetivo de identificar o número de obras públicas paralisadas por achados relacionados ao custo.

$\mathrm{Na}$ segunda etapa foi realizado um estudo do processo de quantificação e orçamentação proposto pelo ABDI em conjunto com o MDIC por meio da estratégia BIM BR. O objetivo deste estudo foi compreender como deverá ser aplicada a metodologia BIM nas dimensões 4D e 5D e identificar qual a contribuição do BIM na redução de irregularidades.

\section{Resultados e Discussão}

A dificuldade do setor público brasileiro em finalizar obras de diferentes portes e complexidade é frequentemente tema de reportagens e os fatores que contribuem para a paralisação estão diretamente relacionadas ao processo de elaboração do custo estimado. A Fiscobras 2018 identificou 77 empreendimentos de infraestrutura, financiadas total ou parcialmente com recursos da União, que apresentaram achado de indício de irregularidade. Conforme as classes definidas pela Lei de Diretrizes Orçamentárias (LDO) esses achados foram classificados de acordo o grau de gravidade, Quadro 1.

Quadro 1 - Classificação das obras conforme grau de gravidade de achados.

\begin{tabular}{|c|l|}
\hline Sigla & \multicolumn{1}{c|}{ Classificação } \\
\hline IGP & Indícios de irregularidade grave com recomendação de paralisação \\
\hline pIGP & $\begin{array}{l}\text { Indícios de irregularidades graves classificados preliminarmente para se tornar IGP, mas ainda } \\
\text { pendente de confirmação pelo TCU. }\end{array}$ \\
\hline IGR & Indícios de irregularidade grave com retenção parcial de valores \\
\hline IGC & Indícios de irregularidades que não prejudicam a continuidade da obra. \\
\hline FI & $\begin{array}{l}\text { Falhas / impropriedades: aqueles considerados de gravidade intermediária ou formal e que } \\
\text { ensejam determinação de medidas corretivas }\end{array}$ \\
\hline SR & Fiscalizações sem ressalvas. \\
\hline
\end{tabular}

Fonte: Autores.

Mediante a classificação que o empreendimento recebeu foram filtradas as obras classificadas em IGP, pIGP e IGR. O que resultou em 16 obras com indícios de irregularidades consideradas graves. Como objetivo dessa pesquisa é identificar problemas relacionados ao custo da obra e a possível solução pela utilização do BIM 4D e 5D, um novo filtro foi aplicado de forma a considerar somente as obras com achados relacionados a quantificação, orçamento e/ou planejamento.

O número final foi reduzido a um total de 10 empreendimentos que apresentaram ao menos um achado de irregularidade que influencia diretamente o custo final do empreendimento, Quadro 2. O achado recorrente foi o sobrepreço decorrente de preços excessivos frente ao mercado. Ainda foi possível verificar a ocorrência de problemas relacionados a quantitativo inadequados, aditivos e superfaturamento de serviços, insumos e encargos. 
Quadro 2 - Empreendimentos por achado de irregularidade.

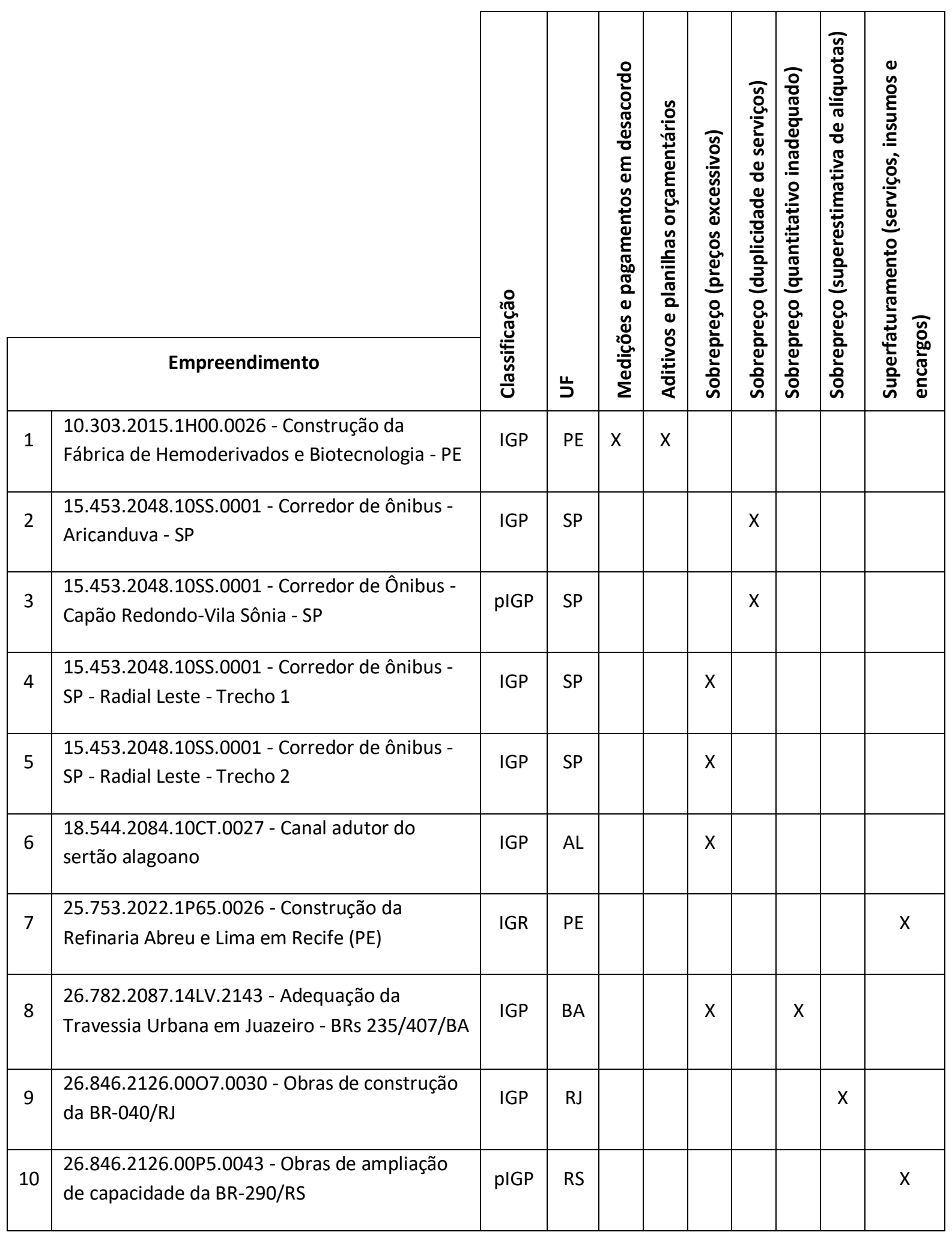

Fonte: Autores.

A aplicação da metodologia BIM 4D para realização do levantamento quantitativo agregado ao BIM 5D para elaboração do orçamento se apresentam como umas possíveis soluções para redução dos achados de irregularidades apontados pela Fiscobras. No GUIA 3 - BIM na Quantificação, orçamentação, planejamento e gestão de serviços da construção, da estratégia BIM BR, é apresentado o processo a ser adotado pelas empresas responsáveis pela aplicação da modelagem 4D e 
$5 \mathrm{D}$.

O processo é dividido em 4 etapas as quais resultaram diferentes quantitativos e relatórios de custos. Isso ocorre pois a cada etapa o projeto (desenho) deve apresentar um maior nível de detalhamento, o que permite a obtenção de informações mais precisas. Desta forma o orçamento final, custo da obra, só é obtido na etapa planejamento quando o projeto atinge o nível de desenvolvimento ND400, Quadro 3.

Quadro 3 - Quadro resumo dos tipos de orçamento e forma de cálculo do preço.

\begin{tabular}{|c|c|c|}
\hline $\begin{array}{c}\text { TIPO DE } \\
\text { ORÇAMENTO }\end{array}$ & $\begin{array}{c}\text { FASE DE } \\
\text { PROJETO }\end{array}$ & CÁLCULO DO PREÇO \\
\hline Estimativa de custo & $\begin{array}{c}\text { Estudos } \\
\text { preliminares }\end{array}$ & Área de construção multiplicada por um indicador. \\
\hline Estudo Preliminar & $\begin{array}{l}\text { Anteprojeto } \\
\text { ND100 }\end{array}$ & $\begin{array}{l}\text { Quantitativos de serviços apurados no projeto ou estimados } \\
\text { por meio de índices médios, e custos de serviços tomados } \\
\text { em tabelas referenciais. }\end{array}$ \\
\hline $\begin{array}{l}\text { Detalhado ou } \\
\text { analítico }\end{array}$ & $\begin{array}{l}\text { Projeto Básico } \\
\text { ND200 }\end{array}$ & $\begin{array}{l}\text { Quantitativos de serviços apurados no projeto e custos } \\
\text { obtidos em composiçôes de custos unitários com preços de } \\
\text { insumos oriundos de tabelas referenciais ou de pesquisa de } \\
\text { mercado relacionados ao mercado local. }\end{array}$ \\
\hline $\begin{array}{l}\text { Detalhado ou } \\
\text { analítico } \\
\text { (orçamento base da } \\
\text { licitação) }\end{array}$ & $\begin{array}{l}\text { Projeto executivo } \\
\text { ND300 }\end{array}$ & $\begin{array}{l}\text { Quantitativos de serviços apurados no projeto e custos } \\
\text { obtidos em composições de custos unitários com preços de } \\
\text { insumos oriundos de tabelas referenciais ou de pesquisa de } \\
\text { mercado relacionados ao mercado local, levando-se em } \\
\text { conta o local, o porte e as peculiaridades de cada obra. }\end{array}$ \\
\hline $\begin{array}{l}\text { Detalhado ou } \\
\text { analítico }\end{array}$ & Planejamento & $\begin{array}{l}\text { Quantitativos apurados no projeto e custos de serviços } \\
\text { obtidos em composições de custos unitários com preços de } \\
\text { insumos negociados, ou seja, advindos de cotações de } \\
\text { preços reais feitas para a própria obra ou para outra obra } \\
\text { similar ou, ainda, estimados por meio de método de custo } \\
\text { real específico. }\end{array}$ \\
\hline
\end{tabular}

Fonte: Autores.

Na etapa do estudo preliminar é possível associar componentes do BIM a indicadores externos obtendo assim um orçamento estimativo de custos e prazo. Estes índices podem ser de custo por unidade de medida $\left(\mathrm{m}, \mathrm{m}^{2}, \mathrm{~m}^{3}\right)$ ou por padrão de acabamento (alto, médio, baixo) do empreendimento. Na etapa do projeto básico são modelados diferentes cenários para um mesmo empreendimento. E a partir destes diferentes cenários são analisados os resultados financeiros e definido as melhorias necessárias do projeto.

Contudo a estimativa de custo e prazo permanece igual ao adotado na etapa do estudo preliminar. Conforme o Decreto n \% 7.983, de 8 de abril de 2013, para obras públicas, os índices a serem utilizados são de bases públicas, repositórios de conhecimento das quais destacam-se o Sistema Nacional de Pesquisa de Custos e Índices da Construção Civil (SINAPI2), operado pela Caixa Econômica Federal (CEF), o Sistema de Custos Referenciais de Obras (SICRO3) para obras de serviços de engenharia, e o Departamento Nacional de Infraestrutura de Transportes (DNIT) para serviços e obras de infraestrutura de transporte.

Com o avançar do detalhamento do projeto, na etapa projeto executivo Quadro 3, são extraídos dados paramétricos e o levantamento de quantitativos gerais necessários para desenvolvimento do orçamento e o planejamento. Assim ao final da etapa de planejamento é gerado o orçamento analítico ou detalhado que a ser utilizado como orçamento base das licitações. 
A precisão do levantamento quantitativo de uma obra é a base de qualquer orçamento ou estimativa de custo. A ABDI no guia sugere alguns procedimentos de extração dos quantitativos dos modelos BIM. As formas de extração variam conforme o aplicativo e metodologia de modelagem que cada empresa irá adotar.

Para a aplicação do modelo 5D, que consiste em vincular as quantidades extraídas a composições de serviços oriundos de um repositório, a ABDI sugere a utilização de softwares capazes realizar a integração do custo ao modelo 4D. Autodesk Navisworks e o Vico Office são os softwares sugeridos pela ABDI como melhores, pois possuem um maior número de funcionalidades. Contudo esta vinculação não é automatizada, pois um determinado serviço pode possuir mais de um tipo de composição, e a escolha da composição correta dependerá da finalidade do orçamento.

\section{Considerações Finais}

Historicamente, grandes projetos de construção têm sido afetados por excedentes de custo e cronograma (Flyvbjerg; Holm; Buhl, 2002). As diferenças entre as estimativas iniciais dos custos do projeto e os preços das propostas ou o custo final podem ser significativas. Ao longo do tempo entre o início do projeto (desenvolvimento do conceito) e a conclusão da construção, muitos são os fatores que podem influenciar no custo final. (Shane et al., 2009)

Durante esta pesquisa foi possível identificar que de 16 obras públicas que se encontravam paralisadas em 2018 por grave indícios de irregularidades, 10 apresentavam achados como sobrepreço e superfaturamento. O processo de aplicação da modelagem BIM 4D e 5D proposto pela ABDI na estratégia BIM BR apresenta pontos positivos que proporcionará aumento da assertividade nas etapas de concepção e gerenciamento das obras. O que promove a redução de irregularidades na gestão, o aumento na transparência das etapas do ciclo de vida da edificação facilitando a fiscalização por órgãos públicos como o TCU.

Outra mudança significativa observada no processo é a utilização de informações oriundas do projeto executivo para elaboração do orçamento analítico que passará a ser utilizado como orçamento base dos editais de licitação. Diferentemente do praticado atualmente onde o orçamento base é extraído do projeto básico o que leva à imprecisão na estimativa de custos e a necessidade de aditivos (Brasil, 2018b).

No entanto ABDI salienta que no mercado brasileiro, as soluções 5D são relativamente restritas. Algumas delas executam somente parte do processo e não oferecem funcionalidades para execução de todo o processo (Brasil, 2017). Eastman et. al (2011) reforçam que o BIM é somente um ponto de partida para a orçamentação. Dificilmente um software conseguirá extrair na totalidade um orçamento de um modelo BIM.

A principal contribuição do trabalho é a verificação de que o processo de quantificação e orçamentação proposto pela ABDI está direcionado ao aumento da precisão, transparecia e confiabilidade por meio de troca de informações entre os diversos profissionais envolvidos em um projeto. Esse direcionamento está alinhado as recomendações e sugestões apresentadas pelo TCU no relatório Diagnóstico das obras paralisadas: Identificação das principais causas e das oportunidades de melhoria, recomendações e monitoramento (Brasil, 2018b).

Para estudo futuro sugere-se discutir como será a interação do conceito BIM, em especial a modelagem 5D, na composição do Benefício e Despesas Indiretas (BDI). Durante está pesquisa foram identificados achados indícios de irregularidades relacionados a superestimativa de alíquotas e encargos. Fatores esses que integram a composição do BDI que não foi abordada no processo proposto pela ABDI.

\section{Referências}

Alaloul, W. S., Liew, M. S., Zawawi, N. A. W. A., \& Mohammed, B. S. (2018). Industry revolution IR 4.0: future opportunities and challenges in construction industry. In MATEC web of conferences. 203, 02010. EDP Sciences.

Brasil. (2017). BIM na Quantificação, orçamentação, planejamento e gestão de serviços da construção: Coletânea Guias BIM ABDI-MDCI. Agência Brasileira de Desenvolvimento Industrial. 
Research, Society and Development, v. 10, n. 1, e25210111681, 2021

(CC BY 4.0) | ISSN 2525-3409 | DOI: http://dx.doi.org/10.33448/rsd-v10i1.11681

Brasil. (2018a). BIM BR - Construção Inteligente. Ministério da Indústria, Comércio Exterior e Serviços.

Brasil. (2018b). Diagnóstico das obras paralisadas: Identificação das principais causas e das oportunidades de melhoria, recomendações e monitoramento. Tribunal de Contas da União.

Eastman, C. M., Eastman, C., Teicholz, P., Sacks, R., \& Liston, K. (2011). BIM handbook: A guide to building information modeling for owners, managers, designers, engineers and contractors. John Wiley \& Sons.

Flyvbjerg, B., Holm, M. S., \& Buhl, S. (2002). Underestimating costs in public works projects: Error or lie? Journal of the American planning association, 68(3), 279-295

Fragoso, R. (2018). Indústria 4.0: Preparados para revolução? https://www.youtube.com/watch?v=DL-DS9A8nvE

Kamardeen, I. (2010, September). 8D BIM modelling tool for accident prevention through design. In 26th annual ARCOM conference. 1, 281-289. Leeds: Association of Researchers in Construction Management.

MC Grawh, C. (2014) The Business Value of BIM for Construction in Major Global MarketsSmartMarket Report. https://www.icnsolutions.nl/pdf/bim_construction.pdf

Miettinen, R., \& Paavola, S. (2014). Beyond the BIM utopia: Approaches to the development and implementation of building information modeling. Automation in construction, 43, 84-91.

Miyasaka, E. L., Minto Fabricio, M., \& Paoletti, I. (2018). Industry 4.0 and the Civil Construction in Brazil.

Nowotarski, P., \& Paslawski, J. (2017, October). Industry 4.0 concept introduction into construction SMEs. In IOP Conference Series: Materials Science and Engineering, 245(5), 052043.

Nardelli, E. S. (2018). BIM and Public Bidding in Brazil, 1212-1219.

Shane, J. S., Molenaar, K. R., Anderson, S., \& Schexnayder, C. (2009). Construction project cost escalation factors. Journal of Management in Engineering, 25(4), 221-229.

Smith, P. (2014a). BIM \& the 5D project cost manager. Selected Papers From The 27th Ipma (International Project Management Association).

Smith, P. (2014b). BIM implementation-global strategies. Procedia Engineering, 85, 482-492.

Succar, B. (2009). Building information modelling framework: A research and delivery foundation for industry stakeholders. Automation in construction, $18(3), 357-375$ 